\title{
STUDIES ON THE CHARACTERS OF ANTIBIOTIC STREPTOMYCES. IV. ON THE STRAINS WHICH PRODUCE STREPTOTHRICIN-GROUP SUBSTANCES
}

\author{
By YosHIRo OKAMT
}

The National Institute of Health of Japan, Tokyo

Several antibiotic substances produced by various kinds of streptomyces are resembling each others. They are adsorbed by activated carbon from the fermented broth and eluted into acid methanol. They are soluble in water and insoluble in organic solvents. However, they are different in toxicity, antibacterial spectra, and some chemical characters. At first Umezawa et al. (1-4), divided them into two groups, that is, streptomycin and streptothricin-group substances. According to the authors (1), substances of streptothricin-group are in the following characters.

1) They are obtained from the broth of streptomyces.

2) They inhibit widely Gram-positive and Gram-negative bacteria.

3) They are adsorbed by activated carbon, eluted into acid methanol, soluble in water and insoluble in organic solvents such as acetone, ether, benzene and petroleum ether.

4) The streptomycin-fast $E$. coli is almost as susceptible to them as the normal culture.

5) The streptothricin-fast $E$. coli is more resistant to them than the normal culture.

Thereafter Umezawa classified the streptothricin-group substances into two groups, the streptothricin-group A and the streptothricin B. The former, if injected into mice, shows a later appearing toxicity, but not the latter. A strain of $B$. anthracis in Umezawa's laboratory is more resistant to streptothricin-group A than B. subtilis (N. R. R. L. B558), but is rather more susceptible to streptothricin B. Streptothricin-group A substances are produced by various kinds of streptomyces, but streptothricin B is obtained from the broth of strains belonging to $S$. fradiae.

In the present paper characters of strains producing streptothricin-group A substances and streptothricin B are described.

\section{Description of Strains}

\section{Strains 68, 201, U-33, B-3, B-10, B-18, 42-B, A-6, K-4 and $\mathbf{E}-2$, which produce streptothricin-group $\mathbf{A}$}

(A) Microscopical observation.

Aerial mycelium is $0.9-1.1 \mu$ width, well branched and coarse. Numerous spirals are observed. Conidia are oval to sphere (1.0-1.2 $\times 1.0-1.2, \mu)$.

(B) Characters on various media.

1) Synthetic agar; $27^{\circ} \mathrm{C}$.

Growth; thin, spreading, colorless, develops deep into medium. Aerial mycelium; cottony to velvety, at first white, later grayish with lavender tinge, this lavender 
color is often lost during successive cultivation. Reverse; colorless or brownish colored. Soluble pigment; none.

2) Glucose bouillon agar; $37^{\circ} \mathrm{C}$.

Growth; thick, wrinkled, cream colored, develops deep into medium. Aerial mycelium; cottony, sometimes with lavender tinge. Soluble pigment; none. Reverse; brownish orange colored.

3) Nutrient agar ; $37^{\circ} \mathrm{C}$.

Growth; thin, wrinkled, cream colored. Aerial mycelium; white scant. Soluble pigment; brownish orange.

4) Loeffler's serum medium; $37^{\circ} \mathrm{C}$.

Growth; thin, dark cream colored. Aerial mycelium; cottony. Substrate changes to brownish around the growth. Liquefaction; none.

5) Egg medium ; $37^{\circ} \mathrm{C}$.

Growth; thin, cream colored. Aerial mycelium; white. Soluble pigment; none.

6) Starch agar ; $27^{\circ} \mathrm{C}$.

Growth ; transparent, colorless. Aerial mycelium; white with lavender tinge. Soluble pigment; none. Enzymatic zone induced by iodine reaction; 2-3 $\mathrm{mm}$ in 5 days.

7) Potato plug; $27^{\circ} \mathrm{C}$.

Growth; thin, wrinkled, colorless to cream colored. Aerial mycelium; white Substrate changes slightly to brownish.

Table 1. Comparison of the strain 68,201 , U-33, B-3, B-10, B-18, $42 \mathrm{~B}, \mathrm{~A} 6, \mathrm{~K} 4$, and $\mathrm{E} 2$ with $S$. lavaendulae (Waksman \& Curtis):

\begin{tabular}{|c|c|c|}
\hline & $\begin{array}{l}\text { Description in Bergey's Det. } \\
\text { Bact. (1948) } \\
(\quad)=\text { Waksman's description (1919) }\end{array}$ & Description of our strains \\
\hline & $\begin{array}{l}\text { Hyphae coarse, branching, spirals close, } \\
5-8 \mu \text { in diam., conidia oval, } 1.0-1.2 \times \\
1.6-2.0 \mu\end{array}$ & $\begin{array}{l}\text { Hyphae coarse, branching, } 0.9-1.1 \mu \text { in } \\
\text { width. Spirals close, abundant, conidia } \\
\text { oval to sphere } 1.0-1.2 \times 1.0-1.2 \mu \text {. }\end{array}$ \\
\hline Synthetic & $\begin{array}{l}\text { Thin, spreading, colorless growth. } \\
\text { Aerial mycelium cottony, white, be- } \\
\text { coming vinouslavender. (S. P. none). }\end{array}$ & $\begin{array}{l}\text { Thin, spreading, colorless growth. } \\
\text { Aerial mycelium cottony, white, be- } \\
\text { coming grayish with lavender tinge. } \\
\text { S.P. none. }\end{array}$ \\
\hline Plain agar & $\begin{array}{l}\text { Gray, wrinkled growth (S.P. brown, } \\
\text { A.M. none) }\end{array}$ & $\begin{array}{l}\text { Creamy-gray, wrinkled A. M. with in } \\
\text { patches. or none S.P. slightly brown } \\
\text { in patches. }\end{array}$ \\
\hline Potato plug & $\begin{array}{l}\text { Thin, wrinkled, cream to yellowish } \\
\text { growth. (A.M. none, plug turns to } \\
\text { brown.) }\end{array}$ & $\begin{array}{l}\text { Wrinkled, creamy growth. Aerial } \\
\text { mycel. white or none. Plug turns to } \\
\text { brown. }\end{array}$ \\
\hline Gelatin stab & $\begin{array}{l}\text { Creamy to brownish surface growth. } \\
\text { Liq. (S. P. brown or none) }\end{array}$ & $\begin{array}{l}\text { Creamy surface growth. A. M. none. } \\
\text { Liq. slow. S.P., brown or none. }\end{array}$ \\
\hline Starch agar & $\begin{array}{l}\text { Restricted., glistening, transparent } \\
\text { growth. (A. M. lavender.) }\end{array}$ & $\begin{array}{l}\text { Glistening, transparent growth. A. M. } \\
\text { lavender colored. }\end{array}$ \\
\hline Litmus milk & $\begin{array}{l}\text { Cream colored ring. No coag. Pept. } \\
\text { with strong alkaline reaction. }\end{array}$ & $\begin{array}{l}\text { Cream colored ring on surface. No } \\
\text { coag. Pept. with strong alkaline. }\end{array}$ \\
\hline $\begin{array}{l}\text { Nitrates } \\
\quad \text { reduction }\end{array}$ & + & + \\
\hline S.P. & Soluble brown pigment. & Soluble brown pigment. \\
\hline Starch hydrol. & & + \\
\hline Opt. temp. & Aerobic $37^{\circ} \mathrm{C}$ & Aerobic $35-38^{\circ} \mathrm{C}$. \\
\hline
\end{tabular}


8) Carrot plug; $27^{\circ} \mathrm{C}$.

Growth ; abundant, wrinkled, brownish. Aerial mycelium; white powdery, with light pink to lavender tinge. Substrate; unchanged or slightly brownish.

9) Gelatin stab; $20^{\circ} \mathrm{C}$.

Growth; glossy surface growth and mass on the bottom of liquefied portion. Liquefaction; slow. Soluble pigment; none or slightly brownish.

10) Litmus milk; $37^{\circ} \mathrm{C}$.

Growth; cream colored surface ring. Aerial mycelium; none. Coagulation; occurs in 3-5 days and then peptonization. Reaction changes to alkaline.

11) Nitrites are not produced from nitrates.

In Table 1 these characters are compared with the description of $S$. lavendulae shown in Bergey's Determinative Bacteriology (1948): They are almost same each others and there are not important differences. The strains described here should be $S$. lavendulae.

\section{Strain 13A which produces streptothricin-group $\mathbf{A}$}

(A) Microscopical observation.

Aerial mycelium is straight and branching. Straight chains of conidia are observed on the end of aerial mycelium. Scale of conidia is $1.0-1.5 \mu \times 1.0-1.5 \mu$ with cylindrical shape. Spirals are not observed.

(B) Characters on various media.

1) Synthetic agar; $27^{\circ} \mathrm{C}$.

Growth ; at first colorless, later reddish orange. Aerial mycelium ; abundant, cottony, chrome orange colored. Soluble pigment; none. Reverse; yellowish.

2) Glucose bouillon agar; $37^{\circ} \mathrm{C}$.

Growth wrinkled, creamy to orange colored, glossy. Aerial mycelium; none or white patches. Brown soluble pigment is often produced.

3) Nutrient agar; $37^{\circ} \mathrm{C}$.

Growth; wrinkled, dark creamy, glossy. Aerial mycelium; none or thin, grayish white. Soluble pigment; slightly brownish.

4) Blood agar; $37^{\circ} \mathrm{C}$.

Growth; wrinkled, greenish gray, glossy. Aerial mycelium; none. Hemolysis; none or very slight.

5) Loeffler's serum medium; $37^{\circ} \mathrm{C}$.

Growth ; cream colored with brownish tinge. Aerial mycelium; none. Liquefaction; none. Soluble pigment; slightly brownish.

6) Egg medium; $37^{\circ} \mathrm{C}$.

Growth; wrinkled, orange colored. Aerial mycelium; white with pink tinge. Soluble brown pigment is around the growth.

7) Starch agar; $27^{\circ} \mathrm{C}$.

Growth; orange colored. Aerial mycelium; white with pink tinge. Enzymatic zone induced by iodine reaction; $1.5-2.5 \mathrm{~mm}$ in 5 days. 
8) Potato plug; $27^{\circ} \mathrm{C}$.

Growth; elevated, greenish. Aerial mycelium; yellowish white with reddish tinge without margin. Substrate changes to black around the growth.

9) Carrot plug; $27^{\circ} \mathrm{C}$.

Growth; spreading, brownish. Aerial mycelium; white to pink. Substrate changes slightly to brownish.

10) Gelatin stab; $20^{\circ} \mathrm{C}$.

Growth; cream colored mass. Aerial mycelium; none. Liquefaction; weak. Soluble pigment; brownish.

11) Litmus milk; $37^{\circ} \mathrm{C}$.

Growth; cream colored ring with pink tinge on surface. Coagulation starts in 3-4 days and later peptonization. Reaction changes to alkaline.

12) Nitrites produced from nitrates.

As seen in Table 2, above characters resemble the characters of $S$. ruber. Apparent differences between them are the formation of spirals, the color of the growth on synthetic agar and the growth on glucose bouillon agar. Though $S$. ruber forms spirals, they are very few. The difference of the color of the growth is not important because they are same in the basic color tone. Therefore, $13 \mathrm{~A}$ strain is similar to $S$. ruber (Krainsky).

Table 2. Comparison of $13 \mathrm{~A}$ with $S$. ruber (Krainsky).

\begin{tabular}{|c|c|c|}
\hline & $\begin{array}{l}\text { Description in Bergey's Det. } \\
\text { Bact. (1948) } \\
(\quad)=\text { Waksman's description (1919) }\end{array}$ & Description of our strain (13 A) \\
\hline & $\begin{array}{l}\text { Straight, branching mycel., radiating. } \\
\text { A few spirals. }\end{array}$ & $\begin{array}{l}\text { Straight, branching mycel. Spirals are } \\
\text { not observed. }\end{array}$ \\
\hline Synthetic & $\begin{array}{l}\text { Abundant, spreading, red growth. } \\
\text { Aerial mycel. abundant, cottony, } \\
\text { : chro-me-orange. (S.P. none.) }\end{array}$ & $\begin{array}{l}\text { Abundant, spreading colorless to red- } \\
\text { dish-orange growth. A. M. abundant, } \\
\text { cottony, orange. } \\
\text { yellowish. }\end{array}$ \\
\hline Plain agar & $\begin{array}{l}\text { Restricted, elevated, wrinkled, olive- } \\
\text { green growth. (A. M. thin, ash gray. } \\
\text { S. P. brown.) }\end{array}$ & $\begin{array}{l}\text { Wrinkled, creamy, glossy, growth. } \\
\text { A. M. thin, grayish. S.P. brown. }\end{array}$ \\
\hline Glucose agar & $\begin{array}{l}\text { Restricted, abundant, entire, coral-red } \\
\text { growth. (A. M. white with pink.) }\end{array}$ & $\begin{array}{l}\text { Wrinkled abundant, reddish-orange } \\
\text { growth. A. M. none or white patches. }\end{array}$ \\
\hline Potato plug & $\begin{array}{l}\text { Elevated wrinkled, greenish growth. } \\
\text { (A. M. red with yellow margin.) }\end{array}$ & $\begin{array}{l}\text { Elevated greenish, growth. A. M. yel- } \\
\text { lowish white with reddish tinge without } \\
\text { margin. }\end{array}$ \\
\hline Gelatin stab & $\begin{array}{l}\text { Liquefaction, with yellow flakes. (A.M. } \\
\text { none. S. P. brown.) }\end{array}$ & $\begin{array}{l}\text { Abundant, orange growth. A. M. } \\
\text { pinkish. }\end{array}$ \\
\hline Litmus milk & $\begin{array}{l}\text { Dark ring with red tinge. Coag. and } \\
\text { pept. with alkaline reaction. }\end{array}$ & $\begin{array}{l}\text { Pinkish cream ring. Coag. and pept. } \\
\text { with alkaline reaction. }\end{array}$ \\
\hline $\begin{array}{l}\text { Nitrates } \\
\text { reduction }\end{array}$ & + & + \\
\hline S.P. & Brown pigment. & Brown pigment. \\
\hline Starch hydrol. & & + \\
\hline Opt. temp. & $37^{\circ} \mathrm{C}$ & $37^{\circ} \mathrm{C}$ \\
\hline
\end{tabular}




\section{Strain 91 which produces streptothricin-group $\mathbf{A}$}

(A) Microscopical observation.

Aerial mycelium is well branched, spirals are short, conidia are $1.2-1.5 \mu \times 1.6-1.8 \mu$ in diameters with oval shape.

(B) Characters on various media.

1) Synthetic medium; $27^{\circ} \mathrm{C}$.

Abundant white to grayish white colored growth. Aerial mycelium; abundant white. Reverse; yellowish. Soluble pigment; none.

2) Glucose bouillon agar; $37^{\circ} \mathrm{C}$.

Growth; thick, wrinkled, edge is myceloid. Aerial mycelium; grayish white, becoming to brownish. Soluble pigment; none.

3) Nutrient agar; $37^{\circ} \mathrm{C}$.

Growth; glossy, scant, cream colored. Aerial mycelium; white in patches. Soluble pigment; none.

4) Blood agar; $37^{\circ} \mathrm{C}$.

Growth; glossy, with brownish gray tinge. Aerial mycelium; none. Soluble pigment; none. Hemolysis; none.

5) Loeffler's serum medium; $37^{\circ} \mathrm{C}$.

Growth; poor, cream colored. Aerial mycelium; none. Soluble pigment; none. Liquefaction; none.

6) Egg medium; $37^{\circ} \mathrm{C}$.

Growth ; poor. Aerial mycelium; none. Soluble pigment; at first none, later reddish yellow pigment (in 7-10 days).

7) Starch agar; $27^{\circ} \mathrm{C}$.

Growth; thin, transparent with cream colored tinge. Aerial mycelium; none. Soluble pigment; none. Enzymatic zone induced by iodine reaction ; $1-3 \mathrm{~mm}$ width in 5 days.

8) Potato plug; $27^{\circ} \mathrm{C}$.

Growth; wrinkled, brownish orange colored. Aerial mycelium; white, abundant. Plug changes to brown.

9) Carrot plug, $27^{\circ} \mathrm{C}$.

Growth; cream colored. Aerial mycelium; grayish white. Plug unchanged.

10) Gelatin stab; $20^{\circ} \mathrm{C}$.

Growth; cream colored surface growth. Aerial mycelium; none or in patches. Slow liquefaction and mass of growth in liquefied portion. Soluble pigment; none.

11) Milk ; $37^{\circ} \mathrm{C}$.

Growth; cream colored ring with brownish tinge on surface. Coagulation and peptonization start from upper portion. Soluble pigment; none.

12) Nitrites produced from nitrates.

13) No tyrosinase.

Among species described in Bergey's Determinative Bacteriology, $S$. albus is most resembling to this strain. However there are some differences. As shown in Table 3, 
the strain, 91, produces reddish pigment on the egg medium, changes the potato to brown, not to purple and hydrolyzes starch. This strain may be a new species though it fairly. resembles $S$. albus. On this strain we shall again study in future.

Table 3. Comparison of the strain 91 with S. albus (Rossi Doria emend Krainsky) Waksman \& Henrici.

\begin{tabular}{|c|c|c|}
\hline & $\begin{array}{l}\text { Description in Bergey's Det. } \\
\text { Bact. (1948) } \\
(\quad)=\text { Waksman's description (1919) }\end{array}$ & Description of our strain \\
\hline Synthetic & $\begin{array}{l}\text { Vegetative hyphae; branched, } 1 \mu \text { in } \\
\text { diam. A. M. abundant, white hyphae } \\
1.3-1.7 \mu \text { in diam. with ellipsoidal spores } \\
\text { (1 } \mu \text {. long) in coiled chains in lateral } \\
\text { branch of the aerial hyphae. (S.P. } \\
\text { none.) }\end{array}$ & $\begin{array}{l}\text { Vegetative hyphae branched, } 0.8-1.0 \mu \text {. } \\
\text { A. M. abundant white, 0.9-1.2 } \mu \text { in } \\
\text { diam. with oval conidia }(1.2-1.5 \times 1.6- \\
1.8 \mu \text { ) in coiled chains (imcomplete } \\
\text { spiralform). Reverse yellow. S.P. none. }\end{array}$ \\
\hline Glucose agar & $\begin{array}{l}\text { Gray A. M. becoming brownish. (Thick, } \\
\text { wrinkled growth, edge myceloid. S. } \\
\text { P. none.) }\end{array}$ & $\begin{array}{l}\text { Thick, wrinkled growth, edge myceloid. } \\
\text { A. M. grayish-white becoming brownish. } \\
\text { S. P. none. }\end{array}$ \\
\hline Bouillon & $\begin{array}{l}\text { No aerial mycel. but chalky-white } \\
\text { deposit forms on old colonies. A.M. } \\
\text { few white patches, glossy cream } \\
\text { colored, spreading. }\end{array}$ & $\begin{array}{l}\text { Grossy, scant, cream colored growth. } \\
\text { A. M. white in patches. S. P. none. }\end{array}$ \\
\hline Potato plug & $\begin{array}{l}\text { Colonies and A. M. white. (Abundant } \\
\text { much wrinkled, cream colored. Plug } \\
\text { changes purplish.) }\end{array}$ & $\begin{array}{l}\text { Brownishorange wrinkled growth. } \\
\text { A. M. white, plug changes to brownish. }\end{array}$ \\
\hline Carrot plug & $\begin{array}{l}\text { Excellent } \begin{array}{l}\text { growth (cream colored } \\
\text { growth. A. M. thin, white.) }\end{array} \\
\end{array}$ & $\begin{array}{l}\text { Cream colored growth. A. M. grayish- } \\
\text { white. }\end{array}$ \\
\hline Gelatin stab & $\begin{array}{l}\text { Liq. Colonies gray, no S.P. (cream } \\
\text { colored growth. A. M. white patches. } \\
\text { S. P. brown but losts by antificial } \\
\text { cultivating.) }\end{array}$ & $\begin{array}{l}\text { Cream colored growth. A. M. none or } \\
\text { white patches. Slow liq. S. S. none. }\end{array}$ \\
\hline Starch agar & $\begin{array}{l}\text { (Spreading, transparent, A. M. none. } \\
\text { S.P. none.) }\end{array}$ & $\begin{array}{l}\text { Thin, transparent with grayish tinge. } \\
\text { A M. none or scant. S.P. none. }\end{array}$ \\
\hline Milk & $\begin{array}{l}\text { (Brownish surface ring. Coag. none.) } \\
\text { Pept. after coag. Alkaline react. }\end{array}$ & $\begin{array}{l}\text { Brownish cream colored ring. Pept. } \\
\text { after coag. Reaction alkaline. }\end{array}$ \\
\hline Starch hydrol. & - (Broad.) & + \\
\hline $\begin{array}{l}\text { Nitrates } \\
\text { reduction }\end{array}$ & + & + \\
\hline
\end{tabular}

\section{Strain 284 which produces streptothricin-group A}

(A) Microscopical observation.

Well branched aerial mycelium bears short spirals. Conidia are ellipsoidal or oval (1.2-1.6 $\mu \times 1.1-1.4 \mu)$.

(B) Characters on various media.

1) Synthetic agar; $27^{\circ} \mathrm{C}$.

Growth; cream colored. Aerial mycelium; abundant, white. Reverse; brownish. Soluble pigment; none.

2) Glucose bouillon agar; $37^{\circ} \mathrm{C}$.

Growth; thick, wrinkled. Aerial mycelium; white to grayish white. Reverse; slightly brownish. Soluble pigment; none or slightly brownish.

3) Nutrient agar; $37^{\circ} \mathrm{C}$.

Growth; cream colored. Aerial mycelium; white. Soluble pigment; none. 
4) Blood agar ; $37^{\circ} \mathrm{C}$.

Growth; brownish, wrinkled. Aerial mycelium; white patches. Soluble pigment; none. Hemolytic action; strong.

5) Loeffier's medium; $37^{\circ} \mathrm{C}$.

Growth; glossy, cream colored with brownish tinge. Aerial mycelium; white. Liquefaction of coagulated serum; rapid.

6) Egg medium ; $37^{\circ} \mathrm{C}$.

Growth; wrinkled, orange colored with brownish tinge. Aerial mycelium; white in patches. Soluble pigment; none:

7) Starch agar; $27^{\circ} \mathrm{C}$.

Growth; transparent. Aerial mycelium; none. Enzymatic zone induced by iodine reaction in 5 days ; 7-10 $\mathrm{mm}$ out side of the growth.

8) Potato plug; $27^{\circ} \mathrm{C}$.

Growth; cream colored. Aerial mycelium; white. Plug unchanged.

9) Carrot plug; $27^{\circ} \mathrm{C}$.

Growth; at first cream colored, afterwards with grayish tinge, thick. Acrial mycelium; grayish white. Plug unchanged.

10) Gelatin stab; $20^{\circ} \mathrm{C}$.

Growth; surface growth is cream colored with brownish tinge. Aerial mycelium; white in patches. Liquefaction; rapid, mass of growth in liquified portion. Soluble pigment; none or orange colored pigment.

11) Milk; $37^{\circ} \mathrm{C}$.

Growth; cream colored surface ring. Aerial mycelium; none. Coagulation; starts in 4-5 days, thereafter peptonization. Soluble pigment; none. Reaction of substrate; alkaline.

12) Nitrites produced from nitrates.

At first the author noticed that this strain should be $S$. albus, but there seem to be many unsuitable points in this determination. The classification will be discussed in detail in future.

\section{Strain 9 which produces streptothricin-group A}

(A) Microscopical observation.

Straight aerial spore-bearing hyphae. Very few spirals. Conidia spherical; $1.0-1.2 \mu \times$ $1.0-1.2 \mu$.

(B) Characters on various media.

1) Synthetic agar; $27^{\circ} \mathrm{C}$.

Growth ; spreading, colorless. Aerial mycelium ; abundant white with dark yellowish green tinge. Reverse; yellowish brown. Soluble pigment; very slight brownish pigment.

2) Gulcose bouillon agar, $37^{\circ} \mathrm{C}$.

Growth; glossy, dark colored. Aerial mycelium; very scant, but occasionally white: patch aerial mycelium with yellowish tinge. Soluble pigment; reddish brown. 
3) Nutrient agar; $37^{\circ} \mathrm{C}$.

Growth; glossy dark cream colored. Aerial mycelium; white with yellowish tinge in patches. Soluble pigment; slight brownish.

$\therefore$ Blood agar; $37^{\circ} \mathrm{C}$.

Growth; glossy, brownish. Aerial mycellium; none. Hemolysis; observed.

5) Loeffler's serum medium; $37^{\circ} \mathrm{C}$.

Growth; light brownish colored. Aerial mycelium; scant white. Liquefaction ; none.

6) Egg medium; $37^{\circ} \mathrm{C}$.

Growth; light brownish colored. Aerial mycelium; dark white. Soluble pigment; grayish brown.

7) Potato plug; $27^{\circ} \mathrm{C}$.

Growth; wrinkled, brownish colored. Aerial mycelium; none. Plug changed slightly to brownish.

8) Carrot plug, $27^{\circ} \mathrm{C}$.

Growth; dark cream colored. Aerial mycelium; none. Plug unchanged.

9) Gelatin stab ; $20^{\circ} \mathrm{C}$.

Growth; brownish surface growth. Aerial mycelium; none. Deep brown pigment diffuses into unliquefied portion. Liquefaction; weak or medium.

10) Milk; $37^{\circ} \mathrm{C}$.

Growth; thick brownish ring on surface. Aerial mycelium; gray. No coagulation and clearing at first. Coagulation occurs after 10 days' incubation. Reaction; slightly alkaline.

Table 4. Comparison of the strain 9 with S. antibioticus (Waksman \& Woodruff).

\begin{tabular}{|c|c|c|}
\hline & $\begin{array}{c}\text { Description in Bergey's Det. } \\
\text { Bact. (1948) }\end{array}$ & Description of our strain \\
\hline & $\begin{array}{l}\text { Spore-bearing hyphae produced in the } \\
\text { form of straight aerial hyphae. The } \\
\text { conidiophores are arranged in clusters. } \\
\text { No spirals formed. The conidia are } \\
\text { nearly spherical to somewhat elliptical. }\end{array}$ & $\begin{array}{l}\text { Spore-bearing hyphae produced in the } \\
\text { form of straight aerial hyphae. Very } \\
\text { few spirals. Conidia spherical. }\end{array}$ \\
\hline Potato plug & $\begin{array}{l}\text { Folded, brown colored growth with a } \\
\text { thin black tinge. No aerial mycelium. }\end{array}$ & $\begin{array}{l}\text { Much folded, deep brown growth. } \\
\text { A. M. none. }\end{array}$ \\
\hline Carrot plug & $\begin{array}{l}\text { Cream colored to faint brownish } \\
\text { growth. No aerial mycel. No pig. }\end{array}$ & $\begin{array}{l}\text { Dark cream colored growth. A. M. } \\
\text { none. Plug unchanged. }\end{array}$ \\
\hline Gelatin stab & $\begin{array}{l}\text { Dark brown growth on surface with } \\
\text { patches of gray aerial mycel. Dark } \\
\text { pig. produced, which gradually diffuse } \\
\text { into the unliquefied part of the gelatin. } \\
\text { Liquefaction at first very slow, later } \\
\text { rapid. }\end{array}$ & $\begin{array}{l}\text { Brownish growth on surface. A. M. } \\
\text { none. Deep brown pig. diffuse into } \\
\text { the unliquefied part. Liq. weak or } \\
\text { medium. }\end{array}$ \\
\hline Litmus Milk & $\begin{array}{l}\text { Thick brownish ring on surface of } \\
\text { milk. Mouse-gray aerial mycel. with } \\
\text { greenish tinge, growth becomes brown, } \\
\text { especially in the portions adhering to } \\
\text { glass. No reaction change, no coag, } \\
\text { no clearing with whitish sediment at } \\
\text { bottom of tube. Old cultures heavy } \\
\text { growth ring on surface of milk, heavy } \\
\text { precipitation on bottom and liquid } \\
\text { brownish to black in upper portion. }\end{array}$ & $\begin{array}{l}\text { Thick, brownish ring on surface. Gray } \\
\text { aerial mycel. slightly alkaline. No } \\
\text { coagulation. No clearing at first. } \\
\text { Coagulation after } 10 \text { days. }\end{array}$ \\
\hline
\end{tabular}


11) Starch plate; $27^{\circ} \mathrm{C}$.

Growth; dark transparent growth. Aerial mycelium; white with dark yellow tinge.

Soluble pigment; none. Enzymatic zone; $3-4 \mathrm{~mm}$ in 5 days.

12) Nitrites produced from nitrates.

Comparing this description with the description in Bergey's Determinative Bacteriology (1948), this strain seems to resemble S. antibioticus (Waksman \& Woodruff) at the points as seen in Table 4. But we find an important difference between them, that is, arranging in clusters of conidiophores.

\section{Strains 117, 260, 250, and 251 which produce streptothricin $B$}

(A) Microscopical observation.

Straight branching filaments and hyphae, no spirals. Conidia are rod to oval $1.0 \times 1.0 \mu$. Spiral is formed on glycerine synthetic agar.

(B) Charcters on various media.

1) Synthetic agar; $27^{\circ} \mathrm{C}$.

Growth; spreading, colorless to dark yellowish. Aerial mycelium; thick, cottony characteristic sea-shell pink to salmon pink colored. Soluble pigment; none.

2) Glucose bouillon agar; $37^{\circ} \mathrm{C}$.

Growth; glossy, cream colored. Aerial mycelium; none or characteristic colored mycelium in patches. Soluble pigment; none.

3) Nutrient agar; $37^{\circ} \mathrm{C}$.

Growth; brownish yellow. Aerial mycelium; none or white patches. Soluble pigment ; none.

4) Blood agar; $37^{\circ} \mathrm{C}$.

Growth; dark with greenish tinge, glossy. Aerial mycelium; none. Hemolyticaction; poor.

5) Loeffler's medium; $37^{\circ} \mathrm{C}$.

Growth; orange colored. Aerial myceliu $r$; none. Soluble pigment; none. Lique-faction; weak.

6) Egg medium; $37^{\circ} \mathrm{C}$.

Growth; thick, wrinkled, orange colored. Aerial mycelium; white. Soluble pigment; none.

7) Starch plate; $27^{\circ} \mathrm{C}$.

Growth; slightly brownish or colorless. Aerial mycelium; characteristic color. Soluble pigment; none. Enzymatic zone; $4-6 \mathrm{~mm}$ in 5 th day.

8) Potato plug; $27^{\circ} \mathrm{C}$.

Growth; wrinkled, orange colored. Aerial mycelium; cream colored. Plug unchanged.

9) Carrot plug; $27^{\circ} \mathrm{C}$.

Growth; poor, cream colored. Aerial mycelium; none. Plug unchanged.

10) Gelatin stab; $20^{\circ} \mathrm{C}$.

Growth; cream colored growth on the surface and bottom of the liquefied portion. Aerial mycelium; white or none. Soluble pigment; none. Liquefaction; rapid. 
11) Milk; $37^{\circ} \mathrm{C}$.

Growth; cream colored surface ring, Aerial mycelium; none or white paches. Coagulation occurs within 10 days then clear up. Reaction; slightly alkaline.

12) Nitrites is produced from nitrates.

These strains are very resembling to $S$. fradiae. The characteristic color of the aerial mycelium seems to justify that they should be $S$. fradiae though they are different in the reducing effect of nitrate.

Table 5. Comparison of the strains, 117, 260, 250 and 251 with S. fradiae (Waksman \& Curtis).

\begin{tabular}{|c|c|c|}
\hline & $\begin{array}{l}\text { Description in Bergey's Det. } \\
\text { Bact. (1948) } \\
(\quad)=\text { Waksman's description (1919) }\end{array}$ & Description of our strain \\
\hline & $\begin{array}{l}\text { Straight, branching filaments and } \\
\text { hyphae. No spirals. Conidia red to } \\
\text { oval, 0.5-0.7 } \times 1.25 \mu \text { (Spiral forms on } \\
\text { glycerin synthetic agar.) }\end{array}$ & $\begin{array}{l}\text { Same as the left side. Conidia } 1.0 \times \\
1.2 \mu \text {. }\end{array}$ \\
\hline Synthetic & $\begin{array}{l}\text { Smooth, spreading, colorless growth. } \\
\text { A. M. thick, cottony mass covering } \\
\text { surface, sea-shell pink. (S.P. none.) }\end{array}$ & $\begin{array}{l}\text { Spreading, colorless to dark yellowish } \\
\text { growth. A. M. thick, cottony, sea-shell } \\
\text { pink to salmon pink. S. P. none. }\end{array}$ \\
\hline Glucose & $\begin{array}{l}\text { Growth; restricted, glossy, buff colored, } \\
\text { lichenoid margin. (A. M. sea-shell pink.) } \\
\text { (S. P. none.) }\end{array}$ & $\begin{array}{l}\text { Brownish yellow growth, A.M. none } \\
\text { or white patches. S.P. none. }\end{array}$ \\
\hline Potato plug & $\begin{array}{l}\text { Restricted, orange colored growth. } \\
\text { (A. M. cream colored patches.) }\end{array}$ & $\begin{array}{l}\text { Wrinkled, orange colored growth. } \\
\text { A. M. cream colored. }\end{array}$ \\
\hline Gelatin stab & $\begin{array}{l}\text { Cream colored to brownish, dense } \\
\text { growth on liquid medium. (A. M. white } \\
\text { or none. S. P. none.) Liq. + }\end{array}$ & $\begin{array}{l}\text { Cream colored mass on surface and } \\
\text { bottom of the liquefied part. A. M. } \\
\text { white or none S.P. none. Liq. +. }\end{array}$ \\
\hline Milk & $\begin{array}{l}\text { Faint, cream colored ring, coagulated } \\
\text { peptonized, becoming alkaline. }\end{array}$ & $\begin{array}{l}\text { Cream colored ring on surface. Coa- } \\
\text { gulation starts in about } 4-6 \text { days. } \\
\text { Peptonized, becoming alkaline. }\end{array}$ \\
\hline $\begin{array}{l}\text { Nitrates } \\
\text { reduction }\end{array}$ & - (good, often only trace.) & + \\
\hline Starch agar & $\begin{array}{l}\text { Spreading colorless growth. (A. M. sea } \\
\text { shell pink.) }\end{array}$ & $\begin{array}{l}\text { Slightly brownish colorless growth. } \\
\text { A. M. characteristic. S. P. none. }\end{array}$ \\
\hline Starch hydrol. & $25^{\circ} \mathrm{C}$ & $24^{+}-27 \mathrm{C}^{+}$ \\
\hline
\end{tabular}

\section{Discussions}

Among strains of streptomyces which produced the streptothricin-group substances, four strains, 260, 117, 250 and 251 resembled $S$. fradiae and besides produced the streptothricin B which is different from the antibiotics of other strains. These four strains, if their characters are compared with the description of $S$. fradiae, are different from the latter in their liquefaction of coagulated serum and their reduction of nitrates. However, they are similar to the latter in all other points. According to the description in Bergey's book, S. fradiae does not reduce nitrate to nitrite, however, in the description by Waksman in 1919 it reduces nitrates. The reduction of the nitrates does not seem to be an important difference. Also the liquesfaction of the coagulated serum seems to be not so important to distinguish these strains from $S$. fradiae. It can be concluded that these strains belong : to S. fradiae. Afterwards, two strains, O-153 and B2 were isolated and found to produce 
streptothricin B. They also belong to $S$. fradiae. It is very interesting that the antibiotic of $S$. fradiae is different from the antibiotics of other species.

Among 13 strains which produced streptothricin-group A substances, 9 strains were very similar to $S$. lavendulae. Any important difference of these strains from S. lavendulae can not be observed. In an experiment testing the utilization of carbohydrates, as shown in Table 6, the results are almost same as those of Pridham who has shown the untilization of sugars of $S$. lavendulae. However the utilization of sugars suggests that there are some kinds in S. lavendulae. From strains of $S$. lavendulae more than one kind of the antibiotics such as streptothricin, lavendulin, streptothricin IV have been obtained. These antibiotics are very resembling each others and seem to belong to our streptothricin-group A. So it can be concluded that streptothricin-group A is most frequently obtained from S. lavendulae.

Table 6 . The utilization of carbohydrates by strains belonging to $S$. lavendulae.

\begin{tabular}{l|c|c|c|c|c|c|c|c|c}
\hline \hline & 201 & $\mathrm{U}-33$, & $\mathrm{B}-3$ & $\mathrm{~B}-10$ & $\mathrm{~B}-18$ & $42-\mathrm{B}$ & $\mathrm{A}-6$ & $\mathrm{~K}-4$ &. .68 \\
\hline Rhamnose & + & + & + & + & + & + & + & + & + \\
Raffinose & - & - & + & - & + & - & + & - & - \\
Xylose & + & + & - & + & - & - & - & + & - \\
\hline
\end{tabular}

The strain, 91, is similar to $S$. albus described in Bergey's book, however, if it is compared with the description of Waksman (1919), then this strain is different from the latter in producing reddish brown pigment on egg medium, changing potato to brown, weak hydrolysis of starch and the weak proteolytic action in milk. Therefore this strain may be different from $A$. albus described by Waksman in 1919. The reddish brown growth on potato has been observed in the case of $A$. albus var. ochroleucus. The strain 91 is most similar to $A$. albus among the species described in Bergey's book. The strain, 284, seems to be also most resembling to $A$. albus, though several characters do not conform to the latter. It is noteworthy that this strain is more resistant to streptomycin than other strains and it grows on the nutrient agar added with streptomycin at $0.05 \mathrm{mg} / \mathrm{cc}$.

The strain, $13 \mathrm{~A}$, is most similar to $S$. ruber. In the case of $S$. ruber spirals have been observed, but they are very few. So though spirals are not observed in the case of the strain $13 \mathrm{~A}$, it should not be an important difference.

The strain, 9, is found to be most resembling to $S$. antibioticus. However it seems to be different from the latter in the form of spore-bearing hyphae. Besides the production of actinomycin is a specific character of this species. The strain, 9, may be a new species, though it belongs to chromogenic type and resembles $S$. antibioticus.

\section{SUMMARY}

(1) Among 13 strains which produced streptothricin-group A substances, 9 strains belonged to $S$. lavendulae, the one to $A$. albus and the one to $S$. ruber. The other two were resembling to $A$. albus and $S$. antibioticus, though they are different in some important points.

(2) Four strains which produced streptothricin B were $S$. fradiae. 
Here I should like to express my sincere appreciation to Dr. Umezawa, chief of our division, and Prof. T. Sasaki of Hokkaido University for their kind advices and directions.

\section{REFERENCES}

1) Umezawa, H., Hayano, S. and Ogata, Y.: Classification of antibiotic strains of Streptomyces and their antibiotic substances on the bases of their antibacterial spectra. Jap. Med. J., 1, 504 1948.

2) Umezawa, H., Hayano, S. and Takeuchi, T.: Studies on the antibacterial substances from Actinomyces, II. A basic antibiotic substance from several strains of Actinomyces. J. Penicillin (Japan), 1, 134, 1948.

3) Umezawa, H., Dan van Ngu, Takeuchi, T. and Sekizawa, Y.: Studies on the antibiotic substances produced by Actinomyces sp. III. A slightly toxic and basic antibiotic substance from a strain of Actinomyces. J. Penicillin (Japan), 1, 221, 1947.

4) Hayano, 'S.: Studies on the antibiotic substances produced by Actinomyces. IV. J. Penicillin (Japan), 1, 24, 1947.

5) Waksman, S. A. and Woodruff, H. B.: Streptothricin, a new selective bacteriostatic and bactericidal agent. Proc. Soc. Exp. Biol. Med., 49, 207, 1942.

6) Waksman, S. A.; Cultural studies of species of Actinomyces. Soil Sci., 8, 71, 1919. 